\title{
EFEITO DE SUBDOSES DE 2,4-D NA PRODUTIVIDADE DO ALGODÃO E SUSCETIBILIDADE DA CULTURA EM FUNÇÃO DE SEU ESTÁDIO DE DESENVOLVIMENTO ${ }^{1}$
}

\author{
JAMIL CONSTANTIN ${ }^{2}$, RUBENS S. DE OLIVEIRA JÚNIOR ${ }^{2}$, JÚLIO R. FAGLIARI ${ }^{3}$, \\ PAULO H. PAGLIARI ${ }^{4}$, JOÃO G. Z. DE ARANTES ${ }^{4}$, SIDNEI D. CAVALIERI ${ }^{4}$, \\ VAGNER P. FRAMESQUI ${ }^{4}$, DIEGO A. GONÇALVES ${ }^{4}$
}

\begin{abstract}
RESUMO: O objetivo deste trabalho foi avaliar o efeito de subdoses de 2,4-D no desenvolvimento e produtividade da cultura do algodoeiro. Dois experimentos foram conduzidos utilizando-se da variedade IAPAR-95 e o sistema de avaliação por meio de testemunhas duplas. No primeiro experimento, as aplicações foram realizadas no estádio F1 (início do florescimento), aplicando-se dosagens de 0,$84 ; 1,68 ; 3,36 ; 6,72 ; 13,44$ e 26,88 g de equivalente ácido (e.a.) por hectare, equivalentes a derivas de 0,$125 ; 0,25 ; 0,5 ; 1,0 ; 2,0$ e $4,0 \%$ da dose de $670 \mathrm{~g}$ e.a. ha $^{-1}$. No segundo experimento, os tratamentos foram constituídos pela combinação em esquema fatorial de duas doses $\left(6,72\right.$ e 13,44 g e.a. ha $\left.{ }^{-1}\right)$ e três épocas de aplicação (C1, C3/C4 e C6), visando a avaliar a variação da sensibilidade do algodão ao 2,4-D em função de seu estádio de desenvolvimento. Os resultados evidenciaram que doses maiores que $3,36 \mathrm{~g}$ e.a. $\mathrm{ha}^{-1}(0,50 \%)$ aplicadas na fase de florescimento afetaram de forma significativa a produtividade, que a queda dos botões florais foi o sintoma mais importante para a redução da produtividade. No segundo experimento, observou-se que a sensibilidade do algodão caiu drasticamente em função do estádio de desenvolvimento. $\mathrm{O}$ único tratamento que provocou queda significativa de produtividade foi a dose de $13,44 \mathrm{~g}$ e.a. ha $^{-1}$ $(2,0 \%)$ aplicada no estádio C1. Dessa forma, a partir do momento em que as maçãs começam a se formar, a sensibilidade da cultura cai substancialmente.
\end{abstract}

PALAVRAS-CHAVE: herbicidas fenóxicos, fitointoxicação, injúrias.

\section{EFFECT OF SUB-LETHAL DOSAGES OF 2,4-D ON COTTON YIELD AND CROP SUSCEPTIBILITY AS A FUNCTION OF ITS DEVELOPMENT STAGE}

\begin{abstract}
This research was aimed at evaluating the effects of sublethal rates of 2,4-D on cotton development and yield. Two field experiments were carried out with cv. IAPAR-95, using two fold-checks. For the first trial, applications were accomplished at the beginning of cotton flowering (stage F1), by spraying rates of $0.84 ; 1.68 ; 3.36 ; 6.72 ; 13.44$ and $26.88 \mathrm{~g}$ of acid equivalent (a.e.) per hectare, equivalent to drifts of $0.125 ; 0.25 ; 0.5 ; 1.0 ; 2.0$ and $4.0 \%$ of a $670 \mathrm{~g}$ of a.e. $\mathrm{ha}^{-1}$ rate. In the second field trial, treatments were composed by the factorial combination of two rates (6.72 and $\left.13.44 \mathrm{~g}_{\text {a.e. }} \mathrm{ha}^{-1}\right)$ and three stages of crop development at the time the herbicide was applied ( $\mathrm{C} 1, \mathrm{C} 3 / \mathrm{C} 4$ e C6). Analysis of the set of data from the first experiment showed that rates higher than $3.36 \mathrm{~g}$ of a.e. $\mathrm{ha}^{-1}(0.50 \%)$ applied during crop flowering decreased yield, being the floral buds drop the most harmful symptom concerning cotton yield. In the second trial, a strong increase in crop tolerance was observed as plants get older. The only treatment decreasing crop yield was the rate of $13.44 \mathrm{~g}$ of a.e. $\mathrm{ha}^{-1}(2.0 \%)$ applied at $\mathrm{C} 1$ stage. Therefore, cotton sensibility to 2,4-D dropped substantially after boll formation.
\end{abstract}

KEYWORDS: phenoxy herbicides, phytotoxicity, injuries.

\footnotetext{
${ }^{1}$ Trabalho apresentado na forma de pôster no IV International Weed Science Congress (Durban, África do Sul - 20 a 24-6-2004).

${ }^{2}$ Eng $^{\mathrm{o}}$ Agrônomo, Prof. Adjunto, Departamento de Agronomia, Universidade Estadual de Maringá, Maringá - PR, Fone (0XX44) 3261.4407, rsojunior@uem.br, Bolsista CNPq.

${ }^{3}$ Engo ${ }^{\circ}$ Agrônomo, Pós-graduando do curso de Pós-graduação em Agronomia da UEM, Bolsista CNPq.

${ }^{4}$ Graduando em Agronomia, UEM, Maringá - PR, Bolsista CNPq.

Recebido pelo Conselho Editorial em: 3-3-2005
}

Aprovado pelo Conselho Editorial em: 17-5-2006 


\section{INTRODUÇÃO}

A fibra de algodão é a mais cultivada e a mais importante das fibras têxteis. A retomada de crescimento da área plantada com a cultura no Brasil ocorreu na década de 1990, com significativo aumento das áreas cultivadas na região Centro-Oeste. No entanto, a expansão das áreas algodoeiras para os cerrados brasileiros trouxe a necessidade de convívio dessa cultura com áreas já cultivadas com outras culturas, como soja e milho. Uma vez que grande parte dessas áreas é cultivada no sistema de plantio direto, é importante também que se entenda a suscetibilidade da cultura a possíveis derivas decorrentes da aplicação de herbicidas nas culturas vizinhas.

O algodoeiro parece tratar-se de uma das culturas mais sensíveis a baixas concentrações de 2,4-D. Segundo GUEVARA (1998), quando o 2,4-D é absorvido pelo algodoeiro durante a fase de florescimento, as flores se deformam, as brácteas e as pétalas se apresentam fundidas e não produzem cápsulas (frutos). As cápsulas que conseguem desenvolver-se são geralmente deformadas e um ou mais lóbulos não desenvolvem sementes. No entanto, concluiu-se que as deformações nas folhas não foram necessariamente correlacionadas aos danos nas sementes. WALL (1996) estudou a tolerância do algodoeiro a diferentes dosagens de 2,4-D (9,5 a 151,2 g e.a. ha ${ }^{-1}$ ), aplicadas duas semanas após a emergência das plântulas, no estádio de desenvolvimento de quatro a seis folhas verdadeiras. As doses corresponderam a 1,5;3,0;6,0;12,0 e 24,0\% daquelas recomendadas para o controle de plantas daninhas dicotiledôneas em culturas de cereais (630 g e.a. ha ${ }^{-1}$ ). Todas as dosagens avaliadas causaram injúrias, tais como epinastia do caule e do pecíolo das folhas, rachadura e deformação da lâmina foliar, deformação da gema terminal e inibição do crescimento. A redução da área foliar variou de 39 (menor dosagem) a 78\% (maior dosagem). A maior dosagem (151,2 g e.a.ha ${ }^{-1}$ ) causou redução de $100 \%$ na produtividade e a dose de $18,9 \mathrm{~g}$ e.a.ha ${ }^{-1}$ ( $3 \%$ da dose recomendada a campo) causou prejuízo de $44 \%$, sendo o teor de óleo reduzido em $16,5 \%$. O autor afirma que esse estudo demonstra a necessidade de se ter muita cautela na aplicação de herbicidas fenoxiacéticos para controle de plantas daninhas quando houver nas proximidades áreas cultivadas com algodoeiro, que se mostrou muito sensível, mesmo com pequenas doses.

A aplicação de subdoses, simulando deriva, tem sido utilizada por diversos pesquisadores com a finalidade de avaliação dos eventuais efeitos em culturas sensíveis. ALVES et al. (2000) utilizaram essa técnica para estimar os efeitos de deriva de glyphosate e oxyfluorfen sobre plantas de milho, utilizando de 2 a $24 \%$ da dose recomendada. De forma semelhante, EBERLEIN \& GUTTIERI (1994) também estudaram a resposta da cultura da batata à aplicação de subdoses de imazamethabenz, imazethapyr e imazapyr, utilizando de 2 a $50 \%$ da dose recomendada para cada herbicida. SCHROEDER et al. (1983) e WALL \& SMITH (2000) também utilizaram essa técnica para avaliação da sensibilidade de diferentes espécies à deriva de 2,4-D.

O objetivo deste trabalho foi avaliar o efeito de subdoses de 2,4-D no desenvolvimento e produtividade da cultura do algodoeiro, bem como verificar o nível de sensibilidade da cultura em função do estádio de aplicação do 2,4-D.

\section{MATERIAL E MÉTODOS}

Dois experimentos foram conduzidos na cultura do algodão no município de Maringá - PR. O solo da área em questão apresentava pH (água) de 6,8; 17,43 $\mathrm{g} \mathrm{de} \mathrm{C} \mathrm{dm}^{-3} ; 76,0 \%$ de argila; $14,0 \%$ de silte e 10,0\% de areia. Para o primeiro experimento, a semeadura foi realizada em 15-1-2004, utilizando-se da variedade IAPAR-95, com espaçamento de 1,00 m entre linhas e seis sementes por metro. A adubação de plantio consistiu de $280 \mathrm{~kg} \mathrm{ha}^{-1}$ do formulado 05-25-15 (N-P-K). A colheita realizou-se em 14-6-2004. Os demais tratos culturais e fitossanitários foram feitos segundo as recomendações da pesquisa para a cultura do algodão no Paraná (IAPAR, 1993).

No primeiro experimento, os tratamentos foram constituídos pela aplicação das doses de 0,$84 ; 1,68 ; 3,36 ; 6,72 ; 13,44$ e 26,88 g e.a. ha $^{-1}$ de 2,4-D, além das testemunhas duplas sem herbicida. Tais doses equivalem a 0,$125 ; 0,25 ; 0,5 ; 1,0 ; 2,0$ e $4,0 \%$ da dose dos produtos comerciais 
mais comuns, aplicados a 1,0 L ha ${ }^{-1}$ (670 g e.a. ha $\left.{ }^{-1}\right)$. Em todos os tratamentos com herbicida, acrescentou-se Agral a 0,1\% v/v. Foram utilizadas testemunhas duplas adjacentes (CONSTANTIN et al., 2001; FAGLIARI et al., 2001; MESCHEDE et al., 2004) a cada parcela tratada, com a finalidade de avaliar com maior clareza e precisão o efeito dos tratamentos.

Para a aplicação, foi utilizado um pulverizador costal de pressão constante, pressurizado a $\mathrm{CO}_{2}$, pressão de $207 \mathrm{kPa}$, equipado com pontas de jato plano tipo leque $\mathrm{AD} 110.02$, proporcionando volume de calda equivalente a $200 \mathrm{~L} \mathrm{ha}^{-1}$.

As aplicações foram realizadas no início do florescimento do primeiro ramo - estádio $\mathrm{F} 1$, de acordo com a escala proposta por MARUR \& RUANO (2001). No momento da aplicação, o solo apresentava-se úmido, a temperatura e a umidade relativa do ar foram, respectivamente, de $27^{\circ} \mathrm{C} \mathrm{e}$ $84,0 \%$. Em todas as aplicações, foram utilizadas cortinas plásticas protetoras para isolamento de cada parcela, evitando-se qualquer deriva para as parcelas vizinhas.

As dimensões das parcelas experimentais foram de 4,0 x 5,0 m, compreendendo quatro linhas de plantio, sendo o delineamento experimental o de blocos ao acaso, com quatro repetições. Para efeito de discussão dos dados, a única comparação de interesse foi aquela que confrontou cada tratamento com sua respectiva testemunha. Portanto, para a análise dos resultados, utilizou-se apenas do teste $\mathrm{F}$, pois o único objetivo do trabalho foi avaliar o grau de injúria de cada tratamento à produtividade da cultura. Foram realizadas avaliações periódicas na cultura após a aplicação dos tratamentos, acompanhando-se a evolução dos sintomas e o efeito sobre o crescimento e floração/frutificação das plantas. A produtividade foi estimada colhendo-se manualmente os $12 \mathrm{~m}^{2}$ centrais de cada parcela.

Após o deslintamento mecânico de amostras de $1 \mathrm{~kg}$ de algodão em caroço por parcela, amostras de sementes foram submetidas à análise de resíduos. Foram utilizadas $10 \mathrm{~g}$ de amostra previamente moída e homogeneizada em frasco Schott de extração. Adicionaram-se $50 \mathrm{~mL}$ de extrator metanol alcalino (Metanol/ $\mathrm{NaOH} 20 \%$ ), passando, a seguir, as amostras no polytron por 30 segundos. As amostras foram agitadas por $2 \mathrm{~h}$ e centrifugadas por 5 minutos a $2.500 \mathrm{rpm}$. Pipetou-se uma alíquota de $10 \mathrm{~mL}$ da solução, que foi evaporada por aproximadamente 90 minutos no turbo-evaporador, a $35^{\circ} \mathrm{C}$. Adicionaram-se $10 \mathrm{~mL}$ de água deionizada, $4 \mathrm{~g}$ de cloreto de sódio e $2 \mathrm{~mL}$ de ácido sulfúrico $6 \mathrm{~N}$. A partição foi realizada em $10 \mathrm{~mL}$ de éter etílico, seguindo-se de agitação vigorosa, sendo as amostras centrifugadas por 3 minutos a $900 \mathrm{rpm}$.

A fase éter foi recolhida em tubos de centrífuga e evaporada $35^{\circ} \mathrm{C}$. Adicionaram-se $3 \mathrm{~mL}$ de metanol aos tubos, os quais foram colocados no ultra-som por 10 segundos. Colunas de alumina foram preparadas com $5 \mathrm{~mL}$ de metanol, carregando-as posteriormente com o extrato, que foi eluído com ácido sulfúrico $1 \mathrm{~mol} \mathrm{~m}^{-3}$. O eluto foi recolhido em tubo de centrífuga, acrescentando a este $4 \mathrm{~g}$ de cloreto de sódio e $10 \mathrm{~mL}$ de éter etílico, agitado vigorosamente e centrifugado por 3 minutos a $900 \mathrm{rpm}$. O éter foi transferido para outro tubo e evaporado novamente. Adicionou-se $0,5 \mathrm{~mL}$ de ácido sulfúrico $1 \mathrm{~mol} \mathrm{~m}^{-3} \mathrm{em} \mathrm{n}$-propanol, levando os tubos novamente ao ultra-som e depois ao bloco aquecedor por 30 minutos a $100{ }^{\circ} \mathrm{C}$. Após a retirada do bloco, as amostras receberam $2 \mathrm{~mL}$ de água deionizada e $2 \mathrm{~mL}$ de solução do padrão interno $\left(0,2 \mu \mathrm{g} \mathrm{mL} \mathrm{m}^{-1}\right.$ picloram P.E. em hexano). As amostras foram novamente centrifugadas como anteriormente, e $4 \mu \mathrm{L}$ da amostra foram injetados no cromatógrafo de fase gasosa modelo HP 5890, equipado com detector seletivo de massa (GC/MSD). As condições de funcionamento do cromatógrafo foram as seguintes: temperatura inicial de $70{ }^{\circ} \mathrm{C}$, com elevação de $20{ }^{\circ} \mathrm{C} \mathrm{min}{ }^{-1}$ até $300{ }^{\circ} \mathrm{C}$ por 4 minutos; detector: $280{ }^{\circ} \mathrm{C}$; injetor: $270{ }^{\circ} \mathrm{C}$; tempo de análise: 16,5 minutos. Esse método resultou num limite de detecção de $0,050 \mathrm{mg} \mathrm{kg}^{-1}$, com recuperação média de $83 \pm 3 \%$ do herbicida na validação do método analítico.

Para o segundo experimento, realizado na safra de 2003-2004, os tratamentos foram constituídos pela combinação em esquema fatorial de duas doses: 6,72 e 13,44 g e.a. ha ${ }^{-1}$ (equivalentes à deriva de 1,0 e 2,0\% da dose comercialmente utilizada do produto) e três épocas de aplicação, com a intenção de avaliar a variação da sensibilidade do algodão ao 2,4-D durante seu 
ciclo de desenvolvimento. A semeadura foi realizada em 15-11-2003. A primeira aplicação foi realizada em 20-2-2004, quando $90 \%$ das plantas de algodão se encontravam entre os estádios F10 e $\mathrm{C} 1\left(\mathrm{~T}=30^{\circ} \mathrm{C}\right.$; UR = 86,0\%); a segunda em 6-3-2004, quando a maioria das plantas de algodão se encontrava no estádio $\mathrm{C} 3 / \mathrm{C} 4 \quad\left(\mathrm{~T}=27^{\circ} \mathrm{C}\right.$; UR $\left.=92,0 \%\right)$, e a terceira em 13-3-2004 (algodão no estádio C6; $\mathrm{T}=27{ }^{\circ} \mathrm{C}$ e UR $=95 \%$ ). Todos os estádios de desenvolvimento do algodão foram definidos seguindo as recomendações de MARUR \& RUANO (2001). Foram adotados os mesmos procedimentos do primeiro experimento com relação ao isolamento das parcelas, número de repetições, delineamento experimental, tratos culturais e avaliação da produtividade.

\section{RESULTADOS E DISCUSSÃO}

No primeiro experimento, verificou-se que o algodão, no início do florescimento (estádio F1), demonstrou grande sensibilidade às derivas simuladas de 2,4-D. A produtividade da cultura foi significativamente reduzida a partir de deriva equivalente a 1,0\% (Tabela 1). A queda dos botões florais foi o sintoma que mais colaborou para a redução da produtividade, sendo os demais sintomas considerados como fatores secundários a influenciar na produtividade da cultura.

TABELA 1. Efeito de doses de 2,4-D sobre a produtividade de plantas de algodão (aplicações no início do florescimento - primeiro experimento).

\begin{tabular}{ccc}
\hline $\begin{array}{c}\text { Dose }(\mathrm{g} \text { e.a. ha } \\
\text { deriva estimado em relaçável de à } \\
\text { dose de } 1 \mathrm{~L} \mathrm{ha}^{-1} \text { das }\end{array}$ & \multicolumn{2}{c}{ Produtividade $\left(\mathrm{kg} \mathrm{ha}^{-1}\right.$ de algodão em caroço $)$} \\
\cline { 2 - 3 } $\begin{array}{c}\text { formulações comerciais }(\%) \\
0,84(0,125 \%)\end{array}$ & Na parcela tratada & $\begin{array}{c}\text { Na respectiva testemunha sem } \\
\text { herbicida }\end{array}$ \\
$1,68(0,25 \%)$ & $1.869,75 \mathrm{a}$ & $1.940,50 \mathrm{a}$ \\
$3,36(0,50 \%)$ & $1.507,75 \mathrm{a}$ & $2.015,50 \mathrm{a}$ \\
$6,72(1,00 \%)$ & $1.254,50 \mathrm{a}$ & $1.851,25 \mathrm{a}$ \\
$13,44(2,00 \%)$ & $617,00 \mathrm{~b}$ & $1.635,75 \mathrm{a}$ \\
$26,88(4,00 \%)$ & $802,25 \mathrm{~b}$ & $1.728,00 \mathrm{a}$ \\
\hline
\end{tabular}

As comparações restringem-se às linhas. Dentro da mesma linha, médias seguidas por mesma letra não diferem entre si, pelo teste $\mathrm{F}$ ( $5 \%$ de probabilidade).

Sintomas de encarquilhamento das folhas mais novas se manifestaram a partir de três dias após a aplicação (DAA), assim como o arroxeamento de folhas e caules e epinastia dos pecíolos. Observou-se, ainda, o amarelecimento e necrose de botões florais, com conseqüente queda dos mesmos, a partir dos 14 DAA, principalmente nas doses mais altas de 2,4-D. Na deriva simulada de 4,0\%, ocorreram rachaduras no caule, engrossamento da raiz principal e da base do caule, demonstrando desenvolvimento anormal do sistema vascular. De acordo com GUEVARA (1998), o 2,4-D causa drástico estrangulamento do câmbio, e isso se deve principalmente à inibição da elongação do ramo principal, a qual resulta na diminuição da formação e elongação de nós. Outra característica interessante de intoxicação de 2,4-D foi o branqueamento das nervuras do limbo foliar.

A folha em forma de "pata-de-rã" é descrita como sintoma típico de intoxicação por 2,4-D. Contudo, é comum a ocorrência de interpretações equivocadas quando se trata de associar a época do aparecimento desse sintoma com a época da ocorrência da deriva. Assim, em reclamações de deriva de 2,4-D, relatos de que os sintomas de "pata-de-rã" surgiram poucos dias após a incidência da deriva, são comumente descritos. O que se observou neste experimento, é que esse sintoma ocorre nas novas folhas que saem após a ocorrência da deriva simulada, mas são visíveis inicialmente apenas a partir de 14 DAA, tornando-se mais evidentes apenas a partir de 21 DAA.

Outro fato observado é que o simples aparecimento de sintomas de "pata-de-rã" ou a supressão do limbo foliar pode não ser suficiente para afetar a produtividade do algodoeiro. Essa também é a interpretação de GUEVARA (1998), que relata que a aplicação de 2,4-D feita no 
estádio de florescimento provoca deformações nas folhas que não são, necessariamente, correlacionadas a danos na produtividade da cultura. Dessa forma, a produtividade da cultura só é realmente afetada caso haja queda expressiva dos botões florais. Mesmo nas menores percentagens de deriva, em que há menor intensidade de queda dos botões e a mesma restringe-se à parte superior das plantas, ainda é possível que a produtividade não seja reduzida, uma vez que os botões apicais normalmente não são componentes importantes na produtividade.

A análise de resíduos de 2,4-D demonstrou que as sementes colhidas, independentemente da dose utilizada, não continham resíduos detectáveis até o limite de quantificação do método empregado $\left(0,050 \mathrm{mg} \mathrm{kg}^{-1}\right)$, conforme apresentado na Tabela 2. Esse fato demonstra que níveis de deriva até $4,0 \%$, durante a fase de florescimento, não propiciariam resíduos de 2,4-D que comprometessem a utilização posterior das sementes de algodão.

TABELA 2. Resíduos de 2,4-D nas sementes de algodão colhidas após a aplicação de diferentes doses no estádio de início de florescimento (F1), segundo escala de MARUR \& RUANO (2001).

\begin{tabular}{cc}
\hline $\begin{array}{c}\text { Dose (g e.a. ha } \\
\text { de } 1 \mathrm{~L} \mathrm{ha}^{-1} \text { e nível de deriva estimado em relação à dose }\end{array}$ & Resíduo $\left(\mathrm{mg} \mathrm{kg}^{-1}\right)$ \\
\hline $0,84(0,125 \%)$ & $<0,05$ \\
$1,68(0,25 \%)$ & $<0,05$ \\
$3,36(0,50 \%)$ & $<0,05$ \\
$6,72(1,00 \%)$ & $<0,05$ \\
$13,44(2,00 \%)$ & $<0,05$ \\
$26,88(4,00 \%)$ & $<0,05$ \\
Testemunha sem herbicida & $<0,05$ \\
\hline
\end{tabular}

No segundo experimento, não foram observados quaisquer sintomas de fitointoxicação em caules, pecíolos ou folhas das plantas, independentemente do estádio de aplicação ou do nível de deriva simulado. Mesmo sem a presença de sintomas evidentes, o tratamento de 13,44 g e.a. ha ${ }^{-1}$ $(2,0 \%)$ aplicado no estádio $\mathrm{C} 1$ afetou de forma significativa a produtividade, em função da queda de botões florais (Tabela 3). No entanto, mesmo esse tratamento não afetou as maçãs já formadas, as quais foram afetadas, independentemente do estádio de aplicação ou da dose. A partir do estádio C3/C4, o algodão suportou derivas de 2,4-D de até $13,44 \mathrm{~g}$ e.a. ha ${ }^{-1}(2,0 \%)$ sem ter sua produtividade comprometida. Dessa forma, a partir do momento em que as maçãs começam a se formar, a sensibilidade da cultura ao 2,4-D cai substancialmente.

TABELA 3. Efeito de doses de 2,4-D na produtividade do algodoeiro $(n=4)$.

\begin{tabular}{lcc}
\hline \multirow{2}{*}{$\begin{array}{c}\text { Estádio de Aplicação* e Dose } \\
\left(\mathrm{g} \text { e.a. } \text { ha }^{-1}\right)\end{array}$} & \multicolumn{2}{c}{ Produtividade $\left(\mathrm{kg} \mathrm{ha}^{-1}\right)$} \\
\cline { 2 - 3 } Estádio C1 - dose 6,72 & Na parcela tratada & Na respectiva testemunha sem herbicida \\
Estádio C1 - dose 13,44 & $1.421,88 \mathrm{a}$ & $1.527,34 \mathrm{a}$ \\
Estádio C3/C4 - dose 6,72 & $1.335,94 \mathrm{~b}$ & $1.601,56 \mathrm{a}$ \\
Estádio C3/C4 - dose 13,44 & $1.554,69 \mathrm{a}$ & $1.515,63 \mathrm{a}$ \\
Estádio C6 - dose 6,72 & $1.563,28 \mathrm{a}$ & $1.597,66 \mathrm{a}$ \\
Estádio C6 - dose 13,44 & $1.587,50 \mathrm{a}$ & $1.703,13 \mathrm{a}$ \\
\hline
\end{tabular}

*Estádios, segundo a escala MARUR \& RUANO (2001). As comparações restringem-se às linhas. Dentro da mesma linha, médias seguidas por mesma letra não diferem entre si, pelo teste $\mathrm{F}(\mathrm{p}<0,05)$.

\section{CONCLUSÕES}

O algodão é sensível à deriva de 2,4-D no início do florescimento $(\mathrm{F} 1)$ e, nesse estádio, tolera no máximo 3,36 g e.a. ha ${ }^{-1}(0,50 \%)$ de deriva desse herbicida. 
A partir do estádio $\mathrm{C} 1$, a sensibilidade do algodão ao 2,4-D é reduzida, e a cultura passa a tolerar até $13,44 \mathrm{~g}$ e.a. ha ${ }^{-1}(2,0 \%)$ de deriva.

\section{REFERÊNCIAS}

ALVES, L.W.R.; SILVA, J.B.; SOUZA, I.F. Efeito da aplicação de subdoses dos herbicidas glyphosate e oxyfluorfen, simulando deriva sobre a cultura do milho (Zea mays). Ciência e Agrotecnologia, Lavras, v.24, n.4, p.889-97, 2000.

CONSTANTIN, J.; OLIVEIRA JÚNIOR, R.S.; FAGLIARI, J.R. Emprego de testemunhas duplas adjacentes na avaliação da seletividade de herbicidas aplicados na cultura do milho. Varia Scientia, Cascavel, v.1, n.2, p.61-74, 2001.

EBERLEIN, C.V.; GUTTIERI, M.J. Potato (Solanum tuberosum) response to simulated drift of imidazolinone herbicides. Weed Science, Champaign, v.42, n.1, p.70-5, 1994.

FAGLIARI, J.R.; OLIVEIRA JÚNIOR, R.S.; CONSTANTIN, J. Métodos de avaliação da seletividade de herbicidas para a cultura da cana-de-açúcar. Acta Scientiarum, Maringá, v.23, n.5, p.1229-34, 2001.

GUEVARA, G. Efecto del 2,4-D sobre el algodón. Saenz Peña: Instituto Nacional de Tecnologia Agropecuária. 1998. 12 p. (Boletim Técnico, 12)

IAPAR. Instituto Agronômico do Paraná. Recomendações para a cultura do algodoeiro no Paraná. Londrina: IAPAR, 1993. 71 p. (Informe de Pesquisa, 107).

MARUR, C.J.; RUANO, O. A reference system for determination of cotton plant development. Revista de Oleaginosas e Fibrosas, Campina Grande, v.5, n.2, p.243-7, 2001.

MESCHEDE, D.K.; OLIVEIRA JÚNIOR, R.S.; CONSTANTIN, J.; SCAPIM, C.A. Período anterior à interferência em soja: estudo de caso com baixa densidade de estande e testemunhas duplas. Planta Daninha, Viçosa, MG, v.22, n.2, p.239-46 2004.

SCHROEDER, G.L.; COLE, D.F.; DEXTER, A.G. Sugarbeet (Beta vulgaris L.) response to simulated herbicide spray drift. Weed Science, Champaign, v.31, n.6, p.831-6, 1983.

WALL, D.A. Effect of sublethal dosages of 2,4-D on annual broadleaf crops. Canadian Journal of Plant Science, Morden, v.76, n.1, p.179-85, 1996.

WALL, D.A.; SMITH, M.A.H. Tolerance of common buckwheat (Fagopyrum esculentum) to low rates of 2,4-D and MCPA. Canadian Journal of Plant Science, Morden, v.80, n.2, p.407-10, 2000. 